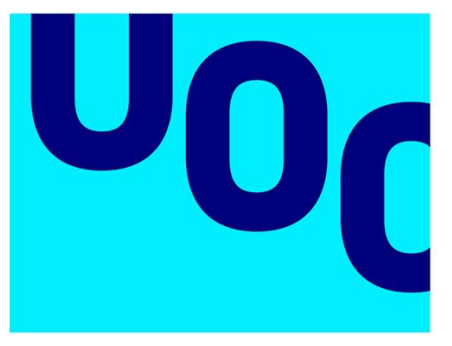

Universitat

Oberta

de Catalunya

\title{
Previsions i realitats de la Realitat Virtual. Evolució 2015-2017
}
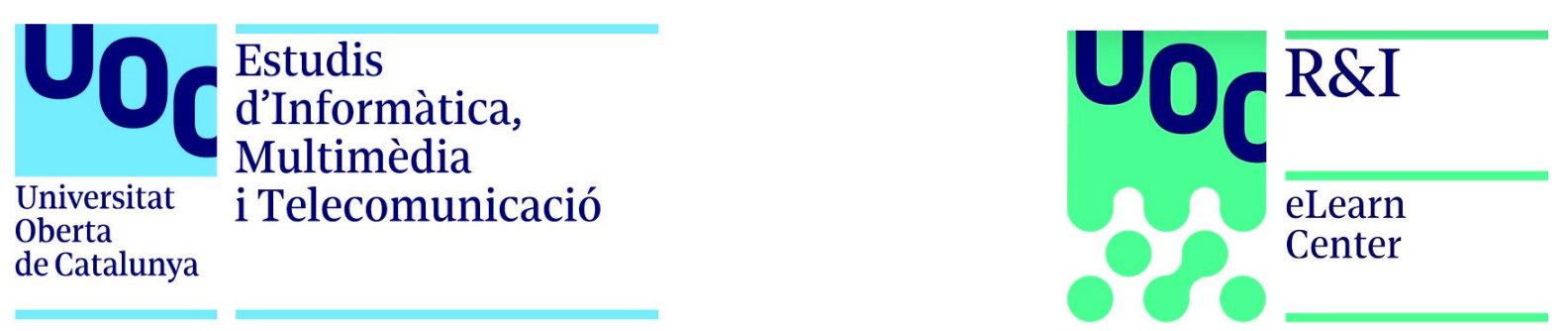


\section{Previsions i realitats de la Realitat Virtual. Evolució 2015-2017}

\section{Crèdits}

Estudis d'Informàtica, Multimèdia i Telecomunicació i eLearn Center. Universitat Oberta de Catalunya.

Coordinadors: Antoni Pérez Navarro i Guillem Garcia Brustenga.

Autors: Robert Clarisó Viladrosa, César Pablo Córcoles Briongos, Desirée Gómez Cardosa, Josep Jorba Esteve, Antoni Pérez Navarro, Laura Vergoñós Pascual i Irma Vilà Òdena.

Edició: Desirée Gómez Cardosa i Laura Vergoñós Pascual.

Citació APA recomanada: Estudis d' Informàtica, Multimèdia i Telecomunicacions (EIMT) i eLearn Center (2018). Previsions i realitats de la Realitat Virtual. Evolució 2015-2017. Barcelona: eLearn Center (UOC).

- ISBN: 978-84-09-07539-3

- DOI: https://dx.doi.org/10.7238/virtual.reality.report.2018

\section{Llicència:}

Creative Commons Attribution 4.0 (CC BY 4.0): https://creativecommons.org/licenses/by/4.0/ Replicate, copy, distribute, transmit, or adapt this report freely as long as that attribution is provided as illustrated in the citation.

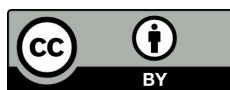




\section{Foreword}

Des dels Estudis d'Informàtica, Multimèdia i Telecomunicació (EIMT) es va engegar el 2014 l'Observatori Tecnològic (OTEIMT), amb l'objectiu de saber quines són les tendències en els àmbits d'interès dels Estudis. L'observatori es va constituir amb representants d'informàtica, de multimèdia, de telecomunicació i d'art (des de la vessant digital), però a més, com que el nucli de la tasca dels EIMT és l'e-Learning d'aquestes disciplines, l'OTEIMT treballa en colllaboració amb l'eLearn Center, que també hi està representat.

En els diversos informes i actes de reunió de l'OTEIMT hi ha hagut un tema que ha anat sortint, el de les realitats virtual (RV), augmentada (RA) i mixta (RM). Sobre aquest tema, les previsions han estat contradictòries sobre quan seria l'any de l'enlairament definitiu d'aquestes tecnologies. Ara bé, el que sembla clar és que aquest enlairament s'acosta gràcies a l'abaratiment i millora tant del maquinari com del programari, però també a la popularització d'algunes aplicacions, tant en l'àmbit professional com lúdic. Analitzar l'evolució de les tres realitats (RV, RA i RM) des de la perspectiva de l'OTEIMT pot ajudar a entendre el perquè els alts i baixos d'aquestes tecnologies.

Antoni Pérez Navarro

Sots-director de Recerca de l'eLearn Center Estudis d'Informàtica, Multimèdia i Telecomunicació

L'eLearn Center, mitjançant el grup de Recerca i Anàlisi de Tendències, té com a objectiu detectar i monitoritzar les tendències pedagògiques, tecnològiques i socials que poden afectar a la UOC a mitjà termini, analitzar-les i fer-ne difusió per tal de posicionar la UOC i l'eLC com a referents en el camp de l'e-Learning. Per tal d' assolir aquest objectiu fem tres activitats clau: observar, analitzar i difondre. Analitzar el que observem és imprescindible per posar en valor i complementar les accions més immediates de divulgació, opinió i curació de continguts. El que donarà rellevància a la feina que fem serà aquesta difusió de coneixement basada en l'anàlisi rigorós, tant cap al públic especialitzat com al públic general. És dins aquest marc on s'ha d'encabir la colllaboració de l'eLC amb els EIMT. La realitat virtual i augmentada és un dels temes analitzats a l'Observatori dels EIMT en els darrers anys, i tothom està d'acord en que pot tenir afectacions importants en l'educació. També és veritat que les RV i RA són una tendència cada any a les previsions de l'educació i que no acaben de concretar-se, possiblement per els alts costos econòmics. Segurament, però, el seu esclat l'educació anirà a remolc dels avenços en altres sectors i, per tant, millor seguir-ne la pista. És per aquesta raó que ens ha semblat molt indicat fer aquest informe de Previsions i realitats de la Realitat Virtual. Evolució 2015-2017.

Guillem Garcia Brustenga

Director del grup de Recerca i Anàlisi de Tendències

eLearn Center 


\section{Introducció}

\section{Definició}

Atès que la Realitat Virtual i la Realitat Augmentada sovint apareixen connectades i que la Realitat Mixta és també un àmbit a l'alça del qual es tracta dins aquest document, es definiran primerament els tres termes.

La Realitat Virtual (d'ara en endavant RV ${ }^{1}$ ) és un entorn d'escenes i/o objectes generat a través de vídeo o videojocs, d'aparença real i on l'usuari s'hi sent immers mitjançant l'ús d'un casc o ulleres creades per a tal finalitat. També es pot augmentar aquesta sensació amb l'ús d'altres gadgets (com per exemple guants) per a percebre més estímuls.

La Realitat Augmentada (d'ara en endavant $\mathrm{RA}^{2}$ ) és la combinació de la visió del nostre entorn real a través d'un dispositiu tecnològic on s'hi afegeix informació virtual en temps real.

La Realitat Mixta (d'ara en endavant $\mathrm{RM}^{3}$ ) és la combinació de RV i RA que permet crear nous espais on interactuen objectes i/o persones reals o virtuals. És una barreja entre realitat, RA, virtualitat augmentada i RV. No s'ha de confondre el terme RM amb el de RA. La RA genera els estímuls a temps real per a la interacció de l'usuari, els quals se superposen sobre l'entorn físic d'aquest; mentre que la RM permet tant la interacció de l'usuari amb l'entorn virtual com que objectes físics de l'entorn immediat de l'usuari serveixin com a elements d'interacció amb l'entorn virtual [75].

\section{Història}

El concepte de Realitat Virtual [1] és molt més llunyà del que a priori podria semblar. El primer registre literari del que es coneix actualment com a RV data del 1935 i pertany a Stanley G. Weinbaum, quan va crear un relat breu de ciència ficció anomenat $\underline{\text { Pygmalion's }}$ Spectacles, que parteix de l'idealisme subjectiu ${ }^{4}$ [114] o l'immaterialisme. La història explica com a través de l'invent del professor Albert Ludwig les persones poden experimentar percepcions sensorials (olor, gust i tacte) a través d'unes ulleres. El relat es desenvolupa entorn de l'espectador de manera interactiva. Cap als anys 50 el tema va tornar a prendre

\footnotetext{
${ }^{1}$ VR (Virtual Reality) en anglès.

${ }^{2}$ AR (Augmented Reality) en anglès.

${ }^{3}$ MR (Mixed Reality) en anglès.

${ }^{4}$ L'idealisme subjectiu afirma que tota realitat es constitueix d'éssers immaterials negant, per tant, l'existència de la matèria.
} 
força, tot i que en aquella dècada es tractava simplement d'il/lustracions i textos referents a màquines capaces de transportar-nos a mons desconeguts d'una realitat alternativa. En aquesta època, però, la tecnologia encara estava lluny de poder fer realitat aquestes idees.

Entre els anys 50 i 70 altres autors van intentar aproximar-se a la idea de la RV a través de la literatura i el teatre, com per exemple el Sensorama de Morton Heilig, que aconsegueix captar tots els sentits de l'espectador a través de la pantalla. L'any 1960 ell mateix patentaria la Màscara telefèrica, definida com "el primer dispositiu de pantalla muntada al cap, que proporciona una televisió estereoscòpica (3D), visió àmplia i veritable so estèreo"[115]. La patent descriu el següent: "S'observa que en col/locar un petit tub de televisió i un sistema de lents de visió perifèrica davant de cada ull de l'usuari, un auricular per cada oïda i un conducte d'aire davant de cada fossa nasal, l'espectador rep una sensació completa de realitat, és a dir: imatges tridimensionals en moviment, que poden ser en color; visió perifèrica 100\%, so binaural, olors i brisa d'aire"[116]. Dins aquesta mateixa època l'empresa Philco Corporation va crear el primer visualitzador amb propòsits d'aprenentatge militar: el Headsight (pantalla i sistema de seguiment connectat a un circuit tancat de TV).

El 1965 Ivan Sutherland va dissenyar l'anomenat Ultimate Display que es va materialitzar el 1968 en forma del primer dispositiu de RV/RA, I'HMD ( Espasa de Dàmocles perquè penjava sobre el cap de l'usuari. Aquest va ser el primer sistema de detecció de l'orientació mitjançant el qual es poden visionar imatges 3D sense moviment. Els gràfics generats per ordinador eren sales i wireframes ${ }^{6}$ molt primitius [117].

El 1975 Myron Krueger va crear el concepte de Realitat Artificial amb el seu projecte de laboratori anomenat Videoplace. En aquest, dues persones en diferents sales, cadascuna amb una pantalla de projecció i una càmera de vídeo, es comunicaven a través de les seves imatges projectades en un «espai compartit» a la pantalla. Cap ordinador va estar involucrat en el primer entorn el 1975. Per realitzar les seves idees sobre una Realitat Artificial, Krueger va començar a desenvolupar el seu propi sistema informàtic fins el 1984, i va haver de dominar els problemes tècnics del reconeixement d'imatges, l'anàlisi d'imatges i la resposta en temps real, i va haver de combinar imatges de vídeo en viu de visitants amb imatges gràfiques, [118]. El projecte ha continuat viu i en transformació al llarg dels anys.

La idea dels entorns virtuals ben aviat es va veure reforçada pels perifèrics estrella: el casc i, posteriorment, els guants. El 1976 es van crear els Sayre Gloves, perfeccionats el 1977 per Daniel J. Sandin i Thomas DeFanti de Electronic Visualization Laboratory, i van crear el

\footnotetext{
${ }^{5}$ Dispositiu muntat al cap.

6 Un algoritme de renderització que conforma un model tridimensional esquemàtic en el qual només es representen línies i vèrtexs.
} 
primer data glove o guant de dades, mitjançant sensors basats en la llum amb tubs flexibles, amb una font de llum en un extrem i una cèl-lula fotoelèctrica a l'altre [120]. El 1981 van aparèixer els primers simuladors de vol controlats per $\mathrm{PC}$, tot i que els guants van ser els perifèrics que més van avançar, amb la creació el 1982 del Data Glove de Thomas Zimmerman. El 1984 el pioner i popularitzador de la RV Jaron Lanier [119], va fundar VPL Research. El 1987, Nintendo va comercialitzar el Nintendo Power Glove, que va apropar aquestes tecnologies al gran públic. VPL Research serà molt important per al desenvolupament de la RV, amb la creació d' enginys com ara l'AudioSphere (crea la il-lusió de sons 3D), l'EyePhone (que utilitza un Data Glove i unes ulleres especials que permeten veure i moure objectes al voltant d'un entorn creat per ordinador) [121], el DataSuit (vestit de cos complet amb sensors per mesurar el moviment de braços, cames i tronc), el Body Electric (llenguatge de programació visual creat per Chuck Blanchard per controlar i programar tots els altres components com a part de tota l'experiència de realitat virtual VPL) i el programa SGI Isaac (escrit per Ethan Joffe, és un motor de renderització visual 3D a temps real controlat pel Body Electric per a crear un entorn virtual) [122]. Lanier i Zimmerman, amb l'ajuda del programador Mitch Altman van crear el Power Glove el 1989.

El 1983 va succeir l'anomenat crash dels videojocs. En el seu apogeu, als Estats Units els ingressos combinats dels arcades i videojocs de consola a la llar era d'uns 11.800 milions de dòlars, cosa que va superar amb escreix els beneficis de les indústries cinematogràfiques i musicals americanes. La indústria va patir una caiguda devastadora: els beneficis obtinguts pels jocs de la consola es van reduir un sorprenent 97 per cent. El 1985, es va estimar que la indústria de la consola, valorada en més de 3.000 milions de dòlars, es valorava aleshores en només 100 milions de dòlars. La causa va ser una suma de factors, com l'arribada sobtada de consoles rivals i els videojocs produïts amb poca cura i pressa, com és el cas del videojoc del film E.T. [123].

La indústria dels videojocs va començar a remuntar a finals dels anys 80 i principis dels 90 , amb la reaparició de dispositius que van tenir una incidència força limitada en el mercat. L'evolució de la RV al llarg d'aquesta època es va centrar sobretot en l'àmbit militar, i va ser així fins a la dècada dels 2000 [131], concretament en l'aprenentatge, a través de simuladors de vol o dispositius mèdics, entre d'altres. També trobem la RV dins la carrera espacial, amb exemples com el d'Antonio Medina, un graduat del MIT i científic de la NASA, que va dissenyar un sistema de RV per a conduir els rovers de Mart des de la Terra, en una mena d'aparent temps real, tot i el substancial decalatge horari als senyals Mart-Terra-Mart [130].

El 1992 es va crear el programari de desenvolupament d'aplicacions de RV WorldToolKit de Sense8 Division of Engineering Animations Inc., fundada per Patrice Gelband i Eric Gullichsen. Consistia en una biblioteca de RV que permetia als desenvolupadors crear 
aplicacions de "món virtual" que funcionaven en ordinadors personals. Primer van aparèixer llançaments per a consoles de saló recreatiu, amb perifèrics i interfaços de RV com ara Dactyl Nightmare de la casa Virtuality. Aquesta empresa va desenvolupar el primer sistema d'entreteniment de RV produït en massa, multijugador i connectat en xarxa. Aquest estava format per múltiples receptacles que constaven de cadires amb auriculars i guants, que van permetre crea la primera experiència immersiva en $\mathrm{RV}$, tot i que a un preu força elevat [125]. La mateixa empresa va desenvolupar el 1995 el Projecte Elysium per a IBM, un sistema de RV per a aplicacions arquitectòniques i de construcció. En quant als primers llançaments comercials de RV per a entreteniment en massa i en format domèstic, Sega va desenvolupar un prototip anomenat Sega $V R$ per a Megadrive, tot i que mai va veure la llum i el projecte es va cancel·lar el $1994^{7}$ [124].

El 1995 es va produir el llançament per a PC del VFX1 de l'empresa desenvolupadora Forte. Consistia en un dispositiu audiovisual en forma de casc amb visor i auriculars, un micròfon, un controlador manual, una placa d'interfície ISA, que oferia rastrejament del moviment del cap, visió estereoscòpica $3 D$ i àudio en estèreo per a ser utilitzat amb videojocs com ara Descent o $\underline{\text { Quake }}^{8}$ [126]. El mateix any va aparèixer el Nintendo Virtual Boy (conegut com a VR-32), la primera consola portàtil 3D amb dispositiu d'ulleres estereoscòpic, tot i que les imatges només es podien veure en vermell i negre i el dispositiu de casc es considerava incòmode [127]. Els esforços de les empreses desenvolupadores començaria aquell any a dirigir-se cap a la col·laboració en línia gràcies a la popularització d'Internet. En paraules d'Albert "Skip" Rizzo9: "Després de 1995, la realitat virtual era prometedora com a tecnologia, però era evident que no estava preparada per jugar. [...] I què més va passar el 1995? Internet. De sobte tothom estava connectat, i la realitat virtual, que era aquest petit germà lleig, quedava enrere" [129].

El 1998 Randy Pausch i Don Marinelli van fundar l'Entertainment Technology Center (ETC) ${ }^{10}$ del Carnegie Mellon, centrat en la educació i el desenvolupament creatiu. A dia d'avui, a través del programa Building Virtual Worlds, la seva agenda de R+D explora els jocs transformacionals, innova en el disseny, treballa la narració interactiva i prepara els estudiants per graduar-se com a professionals creatius [133].

El 1999, l'empresari Philip Rosedale va formar Linden Lab junt amb Andrew Linden per desenvolupar hardware dins el camp dels haptics ${ }^{11} \mathrm{o}$ aparells de funcionalitats tàctils per a

\footnotetext{
${ }^{7}$ Suposadament l'aparell podia causar perjudicis físics amb un ús prolongat.

${ }^{8}$ Per a veure una mostra i conèixer totes les característiques, recomanem el següent vídeo (veure referència [126]).

${ }^{9}$ Director de Realitat Virtual aplicada a la Medicina a l'Institute for Creative Technologies de la University of Southern California i pioner de la RV.

${ }^{10}$ Centre per a la tecnologia de l'entreteniment.

${ }^{11}$ La comunicació hàptica o cinestèsica recrea el sentit del tacte mitjançant dispositius que poden incorporar sensors tàctils per a mesurar les forces, vibracions o moviments de l'usuari, podent ajudar a crear objectes
} 
$\mathrm{RV}$, amb el dispositiu anomenat The Rig, tot i que finalment va sobreeixir més en l'àmbit del software en crear l'entorn virtual Second Life [128].

Skip Rizzo i Mark Bolas ${ }^{12}$ van estar treballant entre el 2002 i el 2006 en la investigació de la RV per a la industria militar. En paraules d'aquest darrer: "L'any 2006 vam presentar aquesta cosa anomenada pantalla Wide Five, i això va canviar tot per a mi. Tenia un camp de visió molt ampli, d'uns 150 graus i podia utilitzar pantalles LCD fora de plataforma. Teníem un contracte amb l'Oficina d'Investigació Naval per trobar una forma de fer RV per a la formació. Bàsicament vam apostar que sorgirien coses com ara els telèfons mòbils i el preu de pantalles més grans cauria" [132].

El 2010 va arribar el primer disseny de les Oculus Rift, sistema que es convertirà posteriorment en referència. A partir d'aquest punt, l'evolució de la RV es comença a desenvolupar de manera exponencial. El 2012 van sortir les Google Glasses, el 2014, Facebook va comprar Oculus Rift per 2 bilions de dòlars, Sony va anunciar el seu projecte Morpheus (un casc per PS4) i Google va anunciar un nou projecte Cardboard mitjançant el qual l'usuari pot construir ell mateix un sistema de RV per al seu telèfon intel-ligent.

\section{Anàlisi del període 2015 - 2017}

Després d'aquest breu repàs a la història de la RV, farem revisarem els darrers 3 anys de RV, RA i RM a través de l'anàlisi dels informes de l'OEIMT per al període 2015-2017. Tot i que els informes es centraven més en RV, en molts casos, no s'ha pogut desvincular de la $\mathrm{RA}$ i de la RM ja que són tecnologies estretament relacionades.

\section{Objectius}

L'ànim ha estat veure, d'una banda, quina ha estat l'evolució d'aquestes tecnologies i com han impactat en les nostres vides; i de l'altra, fins a quin punt s'han fet realitat o no, els pronòstics que se n'havien fet. Per fer aquest informe, s'ha recollit la informació de dues fonts principals: les previsions de tendències de mitjans especialitzats i les notícies i informes de fonts més o menys divulgatives de l'àmbit.

virtuals en una simulació per ordinador, controlar aquests objectes virtuals i potenciar el control remot de màquines i dispositius (telerobòtics). Per a més informació, veure 'Haptic technology', Wikipedia, 2018. [en línia]. [en línia]. Disponible a: https://en.wikipedia.org/wiki/Haptic technology (Consultat el 07/09/2018).

${ }_{12}$ Mark Bolas és president i cofundador de Fakespace Inc. És professor de Mitjans Interactius a la Facultat d'Arts Cinemàtiques de la University of Southern California, Director del seu Laboratori de Narratives Interactives $\mathrm{i}$ Tecnologies Immersives i Director del Laboratori de Realitat Mixta a l'Institut per a les Tecnologies Creatives de la mateixa USC. Actualment es troba en un període d'excedència a la Univesitat per treballar a l'equip d'HoloLens a Microsoft. 


\section{Estructura}

El document s'estructura per anys. Per a cada any es mostra quines van ser les previsions i quina va ser la realitat que es va acabar manifestant, pel que fa a l'aplicació de la RV en diferents àmbits. Al final s'inclou una infografia de resum i un apartat de conclusions.

\section{Previsions del 2015}

Tot i que els inicis de la RV són molt anteriors a l'any 2015, el despuntament d'aquesta tecnologia es produeix a partir de l'any 2014, amb l'arribada de dispositius de RV al gran públic i a uns preus molt més assequibles dels que havien tingut fins al moment.

Dos dels principals mitjans en observació de tecnologies emergents com són l'IEEE [2] i Horowitz [3], recollien en les seves previsions per l'any 2015 la RV com una tecnologia rellevant. En el cas de l'IEEE, la previsió es trobava emmarcada dins la previsió de telèfons més intel-ligents encara i connectats amb sensors, on es parlava de les ulleres de RV.

Gartner [4], tot i posar molt d'èmfasi en els dispositius mòbils intel-ligents (smartphones), parlant de wearables i aplicacions per a aquests dispositius, el 2015 no fa referència a tot el que envolta la RV directament. I en les previsions de la resta de mitjans consultats Frog [5],

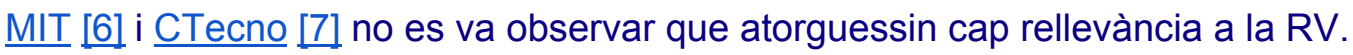

Pel que fa a les previsions de l'OEIMT en el seu informe, la RV va ser considerada "una aposta tecnològica de risc". Els principals problemes que s'hi van detectar eren la manca d'aplicacions i l'acceptació social, i s'hi remarcava la possibilitat que per als anys 2017-2018 la tecnologia hagués despuntat o bé quedat una altra vegada en letargia com ja havia succeït als anys $80-90$.

\section{Realitat l'any 2015}

L'evolució que estava fent tot el món del vídeo i els dispositius mòbils semblava anar encaminada a que els dispositius mòbils enlairessin definitivament la RV. La imatge va guanyar protagonisme de la mà de millores en els sistemes de comunicació i, per tant, en la velocitat de connexió, ajudant a potenciar el vídeo.

El 2015 també va haver expectació per com evolucionarien els aparells. En aquell moment, les previsions de dispositius eren les següents:

- Google Glass [8]: eren ja al mercat però com un producte sense finalitzar que s'havia llançat de manera prematura, sense tenir en compte problemes com ara la privacitat 
i el rebuig que això podia causar [9]. Google tenia previsió d'estendre la RV mitjançant Google Cardboard i la biblioteca de JavaScript per a renderitzar 3D Three.js [10].

- HoloLens: l'aposta de Microsoft [11] [12] que inclou la interacció amb veu mitjançant Cortana [13] [14] i disposen de procesador hologràfic [15]. Tanmateix, encara presenten alguns problemes [16] [17]. Cal destacar que Microsoft estava treballant la part d'aplicacions [18] i estava realitzant proves amb Kinect i RV [19].

- Oculus [20]: la previsió era que apareguessin el primer trimestre de 2016 [21] [22]. Estaven treballant molt el tema de maquinari i noves aplicacions [20] [23].

A banda d'aquestes, la resta de fabricants començava a produir els seus propis aparells: Samsung, HTC, Sony, etc. [24] [25].

\section{Previsions del 2016}

Gartner [26], entre Les 10 tecnologies i capacitats mòbils més importants per al 2015 i 2016, detectava com a tendència Advanced Mobile UX Design, on hi anirien incloses tant la RA com la RV. Cal tenir en compte però, que la llista de Gartner se centra en l'empresa en sí, més que no pas en les tecnologies, per tant, les seves prediccions poden ser complementàries a les dels altres organismes.

Google va incloure el 2016 les RV i RA dins les 6 tecnologies que canviaran el món [27]; i a criteri de Forrester's [30], ambdues estan entre les top 15 tecnologies emergents que cal mirar el 2017-2021. Tanmateix a a l'informe de l'IEEE per al 2022 [28] no es parla de RV, ni tampoc a l'informe del PEW Research Center per al 2025, segons el Digital Life [29], tot i que sí que té en compte els components de la RA, mitjançant l'ús de tecnologies wearables/implantables.

\section{Realitat l'any 2016}

A l'informe de 2015 es va considerar que no era el moment de la RV. Tot i així, diverses declaracions en el Mobile World Congress 2016 i l'aposta per desenvolupar diversos cascos van convertir aquesta tecnologia en una de les recomanacions triades per al 2016. Mark Zuckerberg, per la seva banda, va declarar que no la veia clarament implantada fins 10 anys després. Pel que fa a les notícies tecnològiques, la RV va començar a ser un habitual amb novetats i millores en els dispositius que ja hi havia al mercat. Segons el Wall Street Journal, Google treballava en el seu propi casc de RV [31] i en la millora de l'àudio [32]. Sony treballava amb la Playstation i Nintendo s'hi tornava a posar [33]. També HTC estava generant bona premsa [34] i Intel i Amazon també s'havien afegit al corrent on s'hi havien sumat també altres dispositius [35]. Leap Motion treballava en el touchless feedback haptic 
[36]. Oculus i HTC Vive van sortir al mercat entre el primer i segon quadrimestre. Tot i que el moviment no acabava aquí: els viatges de Google virtual reality esdevenien disponibles per a tothom [37]; Twitter confirmava el llançament d'una divisió de RA [38]; Google i Qualcomm treballaven en el projecte Tango_[39]; i Nvidia treballava per millorar els gràfics i la immersió [40]. A més, Tim Cook d'Apple va dir que la RA és una "core technology" per a la companyia [41] i continuava contractant experts en el tema[42]. En quant a la RV, Cook afirmava que aquesta "no era cosa de nínxol" i que té "aplicacions interessants" [47].

En aquell moment però, calia vigilar els preus, no només dels cascos (de $600 €$ a $800 €$ ) sinó també el de l'equip associat: un PC compatible rondava els $1.500 € \mathrm{i}$ una targeta gràfica compatible sobre $300 €$ més addicionals. En total, calien uns $2.500 €$ per muntar el sistema. Altre tipus de maquinari opcional que calia tenir en compte i que podia acabar de disparar el preu podia ser: per a les Vive, un guant de RV [43] [44]; Sennheiser's va llençar un audio 3D per fer el so de la RV totalment natural [45]; també va aparèixer Nokia Ozo: un sistema de càmera $360^{\circ} 3 \mathrm{D}$ per RV [45b]; i finalment el sistema de Microsoft's hand-tracking magic que podia portar a un sistema de RV més lliure [46].

Alguns exemples de camps d'aplicació que esdevingueren notícia són:

- Google Cardboard es va fer servir per explorar el cor [48].

- La darrera exposició d'Ai Wei Wei a Londres, estava disponible en video immersiu [49].

- Public Speaking" per a la Cardboard ajudava les persones a practicar per parlar en públic [50].

- Es podien mirar Sundance's VR Films en el propi smartphone[51].

- La cadena Fox portava la RV al límit amb 30 minuts a Mart.

- Fox va emetre el bàsquet universitari en $360^{\circ}$ i Facebook va treure la tecnologia perquè hi hagués una adaptació [52].

- Poder participar en una comèdia en RV [53].

- Les HoloLens de Microsoft es feien presents a l'ESA ${ }^{13}$ [137]. L'astronauta de la NASA, Scott Kelly, i l'astronauta de l'ESA, Tim Peake, van provar un nou projecte de RA durant la seva missió a l'Estació Espacial Internacional per ajudar els astronautes a treballar de manera més eficient superposant informacions. Després de dues hores d'experimentar amb el Projecte Sidekick, van trobar un joc amagat: invasors d'espais de RV. El control de la missió els va permetre un moment de diversió com mostra aquest video.

- RV per a fer demos (cases, estands, etc.): Ikea utilitzava la RV d'HTC Vive per ensenyar la cuina abans de comprar-la [54].

- Telepresència via RA (HoloLens de Microsoft) [148].

- KTM va fer una proposta as low cost as it get: el manual de servei en RA [55].

${ }^{13}$ European Space Agency. 
- Amb HoloLens es podia remodelar la cuina a Lowe's [56].

- Sky estava convertint-se en un emisor de RV [57].

També es va detectar cert èmfasi en el vídeo immersiu, per exemple en el cas del fútbol [58], el partit disputat entre el F.C. Barcelona i el Reial Madrid de l'abril de 2016 es va emetre en RV [59]. USA Today va anunciar que hi hauria un show de notícies en RV VRtually There [60]. Anant un xic més lluny, els astronautes van començar a fer trucades d'Skype amb HoloLens [61]. En el camp de l'aeronàutica terrestre, Boeing utilitzava les Google Glass per construir avions [62].

Hi havia diverses iniciatives de desenvolupament de RV: Unity, Unreal Engine, WebVR... [63] Mozilla ha va treure una aplicació de WebVR, VRML (llenguatge de marques de RV). Google obria Daydream, la plataforma de RV per desenvolupadors [64] i Razer llençava el segon OSVR devkit [65].

Les unitats venudes d'Oculus, PS4, etc. en 12 mesos van passar de 300.000 a 500.000 unitats. Un cop llançats al mercat els dispositius d'Oculus i HTC/Steam, semblava que el que havia de seguir era un èmfasi en la RA [66]. Els assistents del 2016 a Coachella van rebre de regal un casc Cardboard VR [67], i també va estar present a la Comic-Con [68].

La premsa també va començar a mostrar cert interès pel que implicava passar gaire temps en entorns de RV, tema en el qual calia més recerca i millores per evitar certs efectes [69]. Durant el 2016 definitivament es va entrar en el que es podria anomenar "fase Second Life": s'havia "d'estar" en el tema per aparèixer als mitjans. També es van començar a considerar els aspectes socials de la RV: Oculus Social va treure al mercat Gear VR, i va arribar al mercat Facebook tie-in [70].

Segons Cara Santa Maria ${ }^{14}$, el que feia increïble el futur de la RV no eren els videojocs o les pel-lícules, sinó la medicina [71]. Tot i així, Epic mirava més enllà dels jocs per nous usos d'Unreal Engine [72].

Relacionat amb l'educació, l'any 2016 es va publicar un article titulat Està preparada la realitat virtual per a l'escola? [79] on s'analitzen les principals barreres per a la seva adopció. Aquestes serien: el cost del maquinari de RV, que en el cas de les escoles públiques podria ser un gran handicap, la manca d'expertesa tecnològica d'alumnes i professorat, la manca de maduresa de les tecnologies de RV i la manca d'encaix amb els currículums establerts.

${ }^{14}$ Cara Santa Maria és periodista de ciència i tecnologia, amfitriona de podcast, reportera de TV guanyadora d'Emmy i co-presentadora de Real Future. 


\section{Previsions del 2017}

A inicis de 2017, pel que fa a les previsions de Gartner [73], seguint l'estela del 2016, la RV i la RA juguen un paper important, tant que entren dins el seu top 10. Per contra, l'IEEE [74] no té en compte aquestes tecnologies. Com ja hem vist, Forrester havia ja inclòs el 2016 la RV dintre de les seves 15 tecnologies emergents que cal mirar el 2017. Microsoft afirmava que les HoloLens encara no es trobarien en disponibilitat per a entorns domèstics [134]. Empreses especialitzades en e-Learning com ara Swift eLearning, destacaven les següents aplicacions de la RA en el sector educatiu: museus, medicina, llibres interactius, entrenament d'habilitats, etc., aventurant que, combinat amb I'm-Learning proveirà experiències en e-Learning sorprenents i engrescadores pels estudiants donat el seu espectre possible d'aplicacions [135]. Dins aquesta línia, el Director de Serveis de Tecnologia Educativa Kyle Bowen de la Penn State University relata com la formació mitjançant aules de RV es fa servir cada cop més a les institucions i creu que les escoles experimentaran amb tecnologia com ara vídeos de $360^{\circ}$ en el futur [136].

\section{Realitat l'any 2017}

Al 2016 vam veure com la RV començava a treure el cap però amb reserves; hi havia un desenvolupament de dispositius, però a preus massa alts per a una butxaca estàndard; a més de la necessitat d'aplicacions, calia solucionar problemes d'usabilitat (com els marejos), entre altres mancances.

Durant el 2017 la RV (i la RA) van prendre un impuls important, i van esdevenir una tendència clau. Han aparegut ulleres més assequibles que acosten aquesta tecnologia al gran públic. Tanmateix, és encara una tecnologia cara, que necessita acabar de madurar i per a la qual encara no trobem la killer application.

També s'ha començat a veure com aplicar aquesta tecnologia en l'àmbit de l'educació, on la RV està començant a entrar. Hem pogut veure algunes notícies que parlen de revolució en l'educació tot i que no es pot parlar encara de canvis generalitzats.

Prova d'això és que a l'Informe OEIMT 2017 [76], quan s'han redactat les tecnologies destacades, la RV ha estat desglossada en diferents apartats, dels quals tractarem en el present document l'estat de la RV, la RM i finalment la simbiosi entre educació i RV.

Segons es va plasmar a l'informe [76b] la informació publicada, es podia preveure que en els propers anys serem testimonis del següent:

- Baixada de preu i potser la capacitat de finançar el maquinari (com ara el telèfon mòbil). 
- 100 millions de dispositius distribuïts, tot i que sense un nombre significatiu d'usuaris, els millors finançadors no es prenen seriosament la construcció de RV sobre el web o el mòbil.

- Nous entorns que facilitin la construcció i integració d'aplicacions de RV.

- Les grans empreses que resolen els principals problemes de maquinari, headsets $\mathrm{i}$ innovació d'entrada, hauran de disposar de més distribució.

La RV és un sector en ple desenvolupament i el personal punter és molt cotitzat. El 2017 Hugo Barra va deixar Xiaomi per executar avenços a la RV de Facebook [82]. El millor dissenyador d'HTC Vive també va passar a treballar a Google Daydream [83].

Les previsions anteriors eren fetes a alguns anys vista, ara repassarem el que ens va aportar el 2017 en quant a novetats.

- Google: la principal plataforma per a RV mòbil continuava sent Daydream amb Daydream View (que ve a ser Cardboard++ amb un comandament), i van anar afegint, lentament, models que el suportaven (Galaxy S8 i S8+ a partir de l'estiu). Es va llençar una línea de cascos standalone (dispositiu integrat dintre de Daydream View, optimitzat, autònom, sense cables ni ordinador a connectar). Es va fer un disseny de referència amb Qualcomm, i HTC i Lenovo preveien treure un producte amb la seva marca. La gran novetat era que els dispositius farien positional tracking: amb la inclusió de dues càmeres (a la presentació deien, literalment, "a handful of sensors', però tot semblava indicar que eren dues càmeres), buscaven punts de referència a l'entorn de l'usuari per a seguir la seva posició amb precisió sense necessitat de càmeres externes (a diferència del que fan les Vive, per exemple). Sobre la política que seguien, semblava correcte el titular de The Verge: "Google no està construint els millors aparells de casc RV. Només la base" [84].

- Samsung: va crear una pantalla per ulleres de RV que triplica els píxels de qualsevol altra [85]. Les vendes de dispositius de casc de RV es van allunyant lentament de les baixades [86] tot i que no es podia assegurar amb claredat si s'accelerava el mercat $\mathrm{o}$ no ja que potser només estava responent a la baixada de preus. Els enviaments a nivell mundial per a cascs de RV d'alta gamma (amb excepcions com Samsung Gear VR i Google Daydream) van superar el milió d'enviaments per primera vegada en el tercer trimestre del 2017, segons l'empresa d'anàlisi de mercats Canalys.

- Apple: els nous iMac incloien el suport a la RV [87].

- Nokia: exempleats de Nokia van prometre una RV amb la resolució de l'ull humà [88].

- ThermoReal: aquesta empresa va pretendre millorar l'experiència de RV aconseguint que l'usuari sentís fred, calor i dolor en jugar [89]. També es va plantejar si hi havia suficient potencial per fer scape rooms de RV [90]. 
El darrer trimestre del 2017 es van vendre 1 milió de dispositius de casc de RV, Sony 49\%, Oculus $21 \%$ i HTC $16 \%$. En especial, s'atribueix la gran pujada de vendes en Oculus a la baixada de preus realitzada [91].

Vam veure com es podien pilotar drons hologràfics i reals amb unes ulleres Epson smart glasses Moverio BTI 300 amb tecnologia hologràfica que permetia veure [92]. Una aerolínia porta ulleres de realitat virtual per treure més informació dels passatgers [93].

Una empresa espanyola, Virtway, va obtenir el récord en usuaris simultanis connectats, fins a 500 persones en una sala 3D virtual per a dispositius mòbils, The Education District [94].

També es va crear una app de realitat virtual per recórrer l'interior d'un gos [95]. De forma sorprenent, Nintendo va anunciar el seu primer joc de RV per HTC Vive: Mario Kart Arcade GP VR [96]. Només 30 apps SteamVR van facturar més de 250 mil dòlars. Gabe Newell ${ }^{15}$ afirmava a una entrevista a Ars Technica: "Si es van agafar els sistemes de RV existents i es van fer un $80 \%$ més barats, això no indica que sigui un mercat enorme. Encara no hi ha una raó molt increïble per a que les persones passin 20 hores al dia en RV". "Una vegada es tingui alguna cosa que realment causi que milions de persones s'emocionin amb la RV, aleshores començarà a haver preocupació per les reduccions de costos. [...] Algunes persones van cridar l'atenció sortint i dient que hi hauria milions de vendes però costa de creure. No es pot indicar cap contingut que causaria que milions de persones canviïn la seva informàtica de casa" [97b].

Google va llençar Blocks per potenciar la creació de contingut en RV. Blocks és una app gratuïta de creació d'objectes per ser mostrats en RV i que permet compartir-ne els resultats per ser aprofitats per altres usuaris. El gran problema de Blocks és que no és per Daydream o Samsung Gear VR, sinó que de moment només estava preparat per ús exclusiu de kits de gamma alta com Oculus Rift y HTC Vive, fet que allunya gran part dels usuaris potencials [98]. Facebook va anunciar que permetria transmetre en viu des la seva app de RV, Spaces, app social de RV presentada per Facebook. Es tracta d'un entorn virtual creat per a Oculus Rift, present a principis del 2017 a la conferència F8 de Facebook. Els usuaris d'Spaces podrien retransmetre en directe en $\mathrm{RV}$, i no només entre ells, també a altres persones sense aquest tipus de tecnologia. La vista del directe no seria la tradicional, sinó que es veuria la imatge flotant a la pantalla [99].

A banda dels anuncis de futurs llançaments i línies de desenvolupament, aquestes van ser algunes de les notícies relacionades amb l'estat actual de la RV en el 2017:

15 Gabe Logan Newell és el co-fundador i director general de l' empresa desenvolupadora de videojocs i distribución digital Valve Software. 
- Hi havia problemes per enfocar bé. El departament de recerca d'Oculus presentava el focal surface display [100]. Havia de millorar la capacitat d'enfocar i ajudar a evitar la nàusea.

- Microsoft Research anunciava el true holographic display [102], [105].

- Nvidia anunciava un holodeck (segons ells, un entorn de RV que complia les lleis de la física) [103].

- Hi havia moviment en eye tracking en RV. HTC Vive podria fer seguiment del moviment ocular amb un upgrade kit de 220 \$ [104].

- Nokia eliminava la seva càmera de RV Ozo i reduïa 310 llocs de treball [106].

En matèria d'educació, la RV podria combinar els millors aspectes de l'aprenentatge de la vida real amb l'aprenentatge en línia en una plataforma integrada. Tres de les maneres en què la RV podria canviar, millorar i fins i tot transformar l'educació superior podrien ser: transformant conferències en experiències d'aprenentatge immersives, permetent als professors realment portar el seu tema a la vida; com a eina de màrqueting per ajudar els estudiants a escollir una universitat; o amb la redefinició de l'ensenyament a distància: en una aula virtual i amb un professor [77]. De fet, es troben articles des de l'any 1992 [78] on ja es relaciona la RV i l'educació tot i que aquest text que ens ocupa se centri en els més moderns.

En el cas dels educadors més pioners, en trobem que utilitzen la RV i la RA a les seves aules amb resultats positius. Un article (Major, 2017) [80] recull algunes aplicacions que es poden utilitzar en l'àmbit de l'educació infantil i universitària per augmentar l'empatia dels alumnes, visualitzar conjunts monumentals, ensenyar ciències, etc. Argumenta sobre la importància dels beneficis que s'estan detectant amb el seu ús, per sobre dels inconvenients. Seguint aquest fil, a l'article Les 15 millors Apps gratuïtes de Realitat Virtual [81] per a l'educació, la idea que s'extreu és que hi ha bones idees en el camp de la RV en Apps per a l'ensenyament però també se'n analitzen les principals mancances:

- Manca d'inversió en el desenvolupament dels gràfics, que sovint són molt pobres.

- La necessitat de la tinença d'ulleres per a acoblar al mòbil fa que el seu ús a classe es vegi reduït o dificultat.

- Algunes de les eines proposades no són realment gratuïtes, sinó demos o, directament s'han de pagar.

- Algunes de les eines atenen més al concepte de videojoc a seques (Cleanopolis VR) o altres recursos que no pas a la educació.

De les eines proposades, l'OEIMT en destaca les següents:

1. History view VR (per utilitzar amb la tecnologia de Merge VR). És molt útil per a l'aprenentatge en entorns museístics.

2. Language VR. Entorn virtual immersiu per a l'aprenentatge de llengües. 
3. Molecule VR. Interessant per a l'aprenentatge del comportament cel-lular i de partícules. Molt poca definició visual.

4. Flashcard VR. Les flashcards poden contenir paraules escrites i imatges i llegir un missatge a l'alumne, ajudant tant a l'aprenentatge visual com a l'auditiu. La idea és bona, però manca inversió i polir la idea.

5. Udacity VR development nanodegree: curs per aprendre a desenvolupar Realitat Virtual. Pot ser una via de futur per als pedagogs i dissenyadors instruccionals.

6. Eon experience VR: Biblioteca de RA i RV amb multitud de recursos educatius.

7. Anatomyou: Aprenentatge de medicina i anatomia en RV. Es fa molt palesa la manca d'inversió en els gràfics.

A continuació desglosarem les novetats en Realitat Mixta. Durant Build, la conferència per a desenvolupadors de Microsoft, es va parlar sobre el tema de la RV fent èmfasi en el concepte de Mixed Reality. La RM es defineix per l'ús de cascos que necessiten un ordinador VR-capable, més o menys de la mida i pes dels d'Oculus i Steam/HTC. Es van mostrar prototips funcionals però de moment només amb demos de RV (l'augmentada hauria d'arribar després, mesclant vídeo que captura el dispositiu amb els grafics generats). Tenien dispositius (HP i Acer) en prevenda per poc més de 300 \$ (controlador a banda, però encara així, extremadament competitius amb els d'HTC/Steam i Oculus, sobre el paper), i la manera de realitzar el positional tracking era molt similar a la dels cascos autònoms de Google[107], [108], [109]. HTC Vive va crear un sistema que permetia que 50 unitats de RV treballessin juntes sense interferències creuades en una escola [110]. Microsoft va desenvolupar un video explicatiu del seu sistema de RM [111] i juntament amb Samsung collaboren en el desenvolupament d'un casc de RM. Tal com revelaven en les imatges filtrades a Twitter, aquestes ulleres de realitat mixta es vendrien amb els motion controllers creats per Microsoft. A més, el dispositiu comptaria amb uns auriculars integrats desenvolupats per l'empresa austríaca AKG, una companyia propietat de Samsung [112]. Intel retallava els seus plans pels dispositius de casc de RM Alloy [113] mentre investigadors de Google Machine Perception en collaboració amb Daydream Labs i YouTube Spaces també experimentaven amb la RM incrustant virtualment el rostre de l'usuari sobre les ulleres de RV [97].

\section{Conclusions}

A través d'aquest informe s'ha pogut fer un recorregut per l'estat de les previsions en matèria de RV, RA i RM de les grans consultores i empreses del sector tecnològic.

L'evolució ha estat ràpida pel que fa a la importància donada a aquestes tecnologies i han passat de ser gairebé residuals i aplicades a uns àmbits reduïts, a formar part integrant de les principals tendències. Els principals àmbits d'inclusió, d'inversió i de previsible aplicació 
són la indústria de l'oci (videojocs, principalment), la cultura (museística i reconstrucció virtual de patrimoni, experiències immersives i creació artística), la medicina (diagnòstic i representació visual durant intervencions quirúrgiques) i l'educació (aules virtuals, pràctiques i laboratoris remots i immersió amb vídeo $360^{\circ}$ entre d'altres).

Les debilitats que hi detectem són: el tarannà de la RV com a tecnologia emergent i en estat de semi-provatura, els problemes de focalització, estabilització i ergonomia del maquinari i la manca d'una popularització a gran escala que permeti el seu abaratiment i ús comú, a més de la gran inversió econòmica per part de les empreses desenvolupadores i creadores. En el terreny de les amenaces, en destacaríem les encara possibles falles de seguretat $\mathrm{i}$ errades de programació, la manca de preus standard per productes de qualitat similar i la possible reticència a adoptar aquestes tecnologies per part d'un públic que encara no hi està acostumat.

D'altra banda, la principal fortalesa tant de la RV com de tecnologies afins és que oferiran oportunitats globalitzadores tant en els diferents àmbits esmentats, com en nous camps que es vagin obrint.

En qualsevol cas, les oportunitats que ofereixen la RV, la RA i la RM són molt diverses, com ara el fet de facilitar processos industrials i intel-lectuals, la creació de nous entorns de treball o les aplicacions que pugui tenir en educació i en la distribució de coneixements. Aquestes tecnologies conformen una personalització de diverses experiències a través de la immersió de l'individu. Estarem pendents de la seva evolució. 


\section{Infografia}

\section{Previsions i realitats de la Realitat Virtual} Evolució 2015-2017

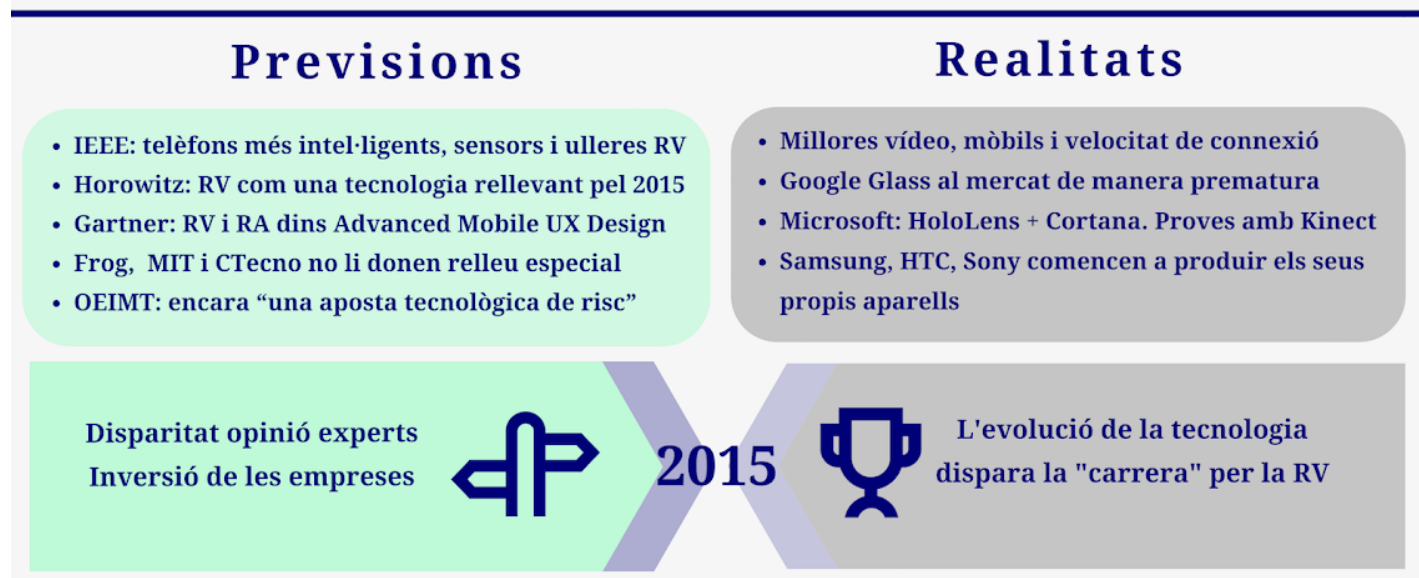

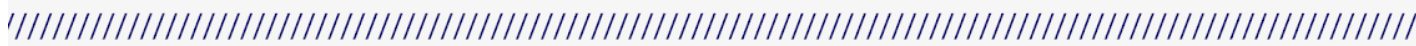

- Google: RV/RA 6 tecnologies que canviaran el món

- Forrester's: RV/RA 15 tecnologies emergents '17-'21

- L'IEEE CS 2022 Report no parla de RV

- PEW Digital Life in 2025: RA + wearables

- OEIMT: RV en puixança. Headsets i auge a MWC '16
- Avenços Google, Intel, Amazon, Unity, Unreal, Mozilla VRML, Razer OSVR dev, Nvidia, Microsoft - Hardware: guant RV Vive, Audio 3D Sennheiser, $360^{\circ}$ 3D VR cam Nokia Ozo. HTC Vive i Oculus al mercat

- Gaming: Sony Playstation i Nintendo

- RV Social: Facebook, Oculus Social, Twitter (RA)

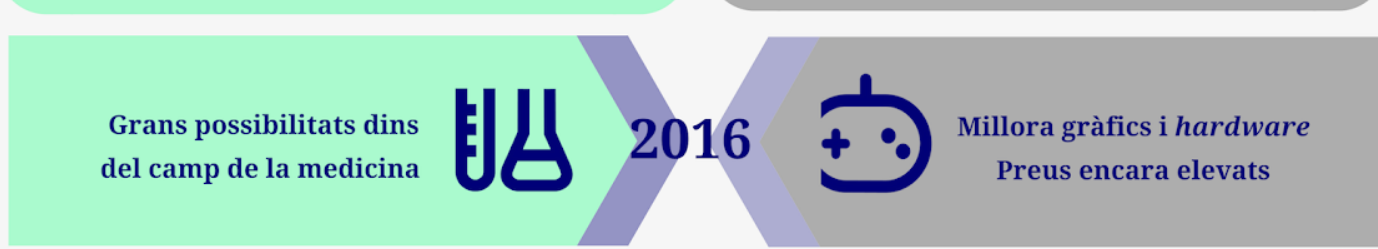

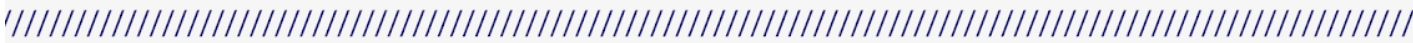

- Gartner: RV i RA dins el top 10

- L'IEEE: no té en compte aquestes tecnologies

- Forrester: RV dins 15 les tecnologies emergents 2017

- HoloLens encara no disponibles a entorns domèstics

- OEIMT: destaca baixada de preu, 100 millions dispositius distribuïts, nous entorns d'aplicació
- Nintendo: 1r joc RV Mario Kart per HTC Vive

- Pilotatge drons hologràfics i reals (ulleres Epson)

- Millores visuals, positional tracking (Oculus,

Samsung, Google, Qualcomm, HTC, Lenovo)

- Treball hologràfic de Microsoft i Nvidia

- Apple: suport RV als nous iMac

- ThermoReal: experiència de fred, calor, dolor
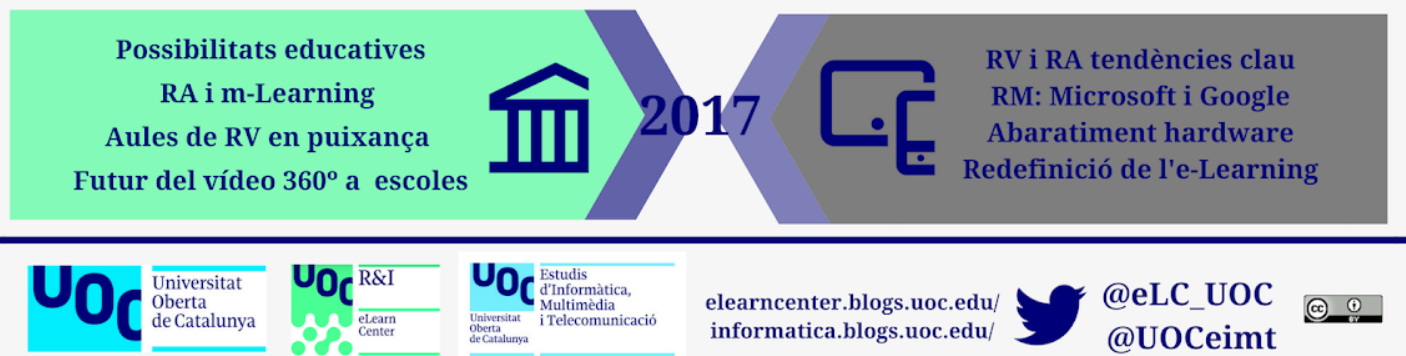


\section{Referències}

[1] 'Historia de la Realidad Virtual' (2018) Wikipedia [en línia]. Disponible a: https://es.wikipedia.org/wiki/Historia de la realidad virtual (Consultat el 20/07/2018).

[2] Neira, E. M. (2015) 'IEEE ComSoc CTN Special Issue on Ten Trends that Tell Where Communication Technologies are Headed in 2015', IEEE CTN [en línia]. Disponible a: https://www.comsoc.org/ctn/ieee-comsoc-ctn-special-issue-ten-trends-tell-where-communica tion-technologies-are-headed-2015 (Consultat el 16/05/2018).

[3] Chokshi, S., Horowitz, A. (2015) '16 Things', Andreessen Horowitz [en línia]. Disponible a: https://a16z.com/2015/01/22/16-things/ (Consultat el 16/05/2018).

[4] Spender, A. (2015) 'Top 10 Strategic Predictions for 2015 and Beyond', Gartner [en línia]. Disponible

https://www.gartner.com/smarterwithgartner/top-10-strategic-technology-predictions-for-2015 -and-beyond/ (Consultat el 16/05/2018).

[5] Allison G. S. et alii. (2015) 'Tech Trends 2015', Frog Design [en línia]. Disponible a: https://www.frogdesign.com/html/techtrends2015/index.html (Consultat el 16/05/2018).

[6] (2015) '10 Breakthrough Technologies 2015', MIT Technology Review [en línia]. Disponible a: https://www.technologyreview.com/lists/technologies/2015/ (Consultat el 16/05/2018).

[7] (2014) 'Baròmetre del Sector tecnològic a Catalunya', Cercle Tecnològic de Catalunya, Barcelona [en línia]. Disponible a: http://www.ctecno.cat/wp-content/uploads/2014/07/TECHNOLOGICAL-SECTOR-BAROMET ER-IN-CATALONIA-2014-ENGLISH.pdf (Consultat el 16/05/2018).

[8] Grau, A. (2015) 'Futuro incierto para las Google Glass', Informàtica ++ [en línia]. Disponible

a:

http://informatica.blogs.uoc.edu/2015/01/29/futuro-incierto-para-las-google-glass/ (Consultat el 28/06/2018).

[9] Gallagher, S. (2015) 'Augmented reality gets to work-and gets past the Glassholes', Ars Technica [en línia]. Disponible a: https://arstechnica.com/information-technology/2015/03/augmented-reality-gets-to-work-andgets-past-the-glassholes/ (Consultat el 28/06/2018).

[10] Catanzariti, P. (2015) 'Bringing VR to the Web with Google Cardboard and Three.js', Sitepoint [en línia]. Disponible a: https://www.sitepoint.com/bringing-vr-to-web-google-cardboard-three-js/ (Consultat el 04/07/2018).

[11] (2015) 'HoloLens. Transform your world with holograms', Microsoft [en línia]. Disponible a: https://news.microsoft.com/videos/microsofts-hololens-demo-at-build-2015/ (Consultat el 04/07/2018).

[12] Warren, T. (2015) 'Microsoft offers a closer look at its HoloLens headset', The Verge [en línia].

Disponible

a: 
https://www.theverge.com/2015/4/30/8521681/microsoft-hololens-headset-features (Consultat el 04/07/2018).

[13] Hamblen, M. (2015) 'Cortana on the PC: Can we talk', Computer World [en línia]. Disponible

https://www.computerworld.com/article/2879473/cortana-on-the-pc-can-we-talk.html (Consultat el 04/07/2018).

[14] Gallagher, S. (2015) 'Windows 10 brings Cortana to the desktop', Ars Technica [en línia].

https://arstechnica.com/information-technology/2015/01/windows-10-brings-cortana-to-the-d esktop/ (Consultat el 04/07/2018).

[15] Hruska, J. (2015) 'Microsoft demonstrates new HoloLens prototype, talks up dedicated Holographic processor', ExtremeTech [en línia]. Disponible a: https://www.extremetech.com/gaming/204763-microsoft-demonstrates-new-hololens-prototy pe-talks-up-dedicated-holographic-processor (Consultat el 04/07/2018).

[16] Bright, P. (2015) 'HoloLens: Still magical, but with the ugly taint of reality', Ars Technica [en línia]. Disponible a: https://arstechnica.com/gadgets/2015/05/hololens-still-magical-but-with-the-ugly-taint-of-reali tyl (Consultat el 04/07/2018).

[17] Robertson, A. (2015) 'Microsoft's HoloLens is new, improved, and still has big problems', The Verge [en línia]. Disponible a: https://www.theverge.com/2015/5/1/8527645/microsoft-hololens-build-2015-augmented-reali ty-headset (Consultat el 04/07/2018).

[18] Lee, N. (2015) 'Microsoft helped me build a HoloLens app, and it was pretty easy

2, Engadget [en línia]. Disponible a:
https://www.engadget.com/2015/05/01/hololens-hands-on/ (Consultat el 04/07/2018).

[19] Wilson, A., Lower, B. (2015) 'Hacking Augmented Reality with Kinect', Channel 9 [en línia]. Disponible a: https://channel9.msdn.com/Events/Build/2015/3-87 (Consultat el 04/07/2018).

[20] Orland, K. (2015) 'This is the PC hardware you'll need to run the Oculus Rift', Ars

Technica [en línia]. Disponible a:
https://arstechnica.com/gaming/2015/05/this-is-the-pc-hardware-youll-need-to-run-the-oculu s-rift/ (Consultat el 04/07/2018).

[21] Robertson, A. (2015) 'The finished Oculus Rift is shipping early next year', The Verge [en línia]. Disponible a: https://www.theverge.com/2015/5/6/8557835/oculus-rift-consumer-edition-virtual-reality-relea se-date (Consultat el 04/07/2018).

[22] Goel, V. (2015) 'Oculus to Ship Virtual Reality Headsets in Early 2016', The New York Times [en línia]. Disponible a: https://bits.blogs.nytimes.com/2015/05/06/oculus-to-ship-virtual-reality-headsets-in-early-201 6/ (Consultat el 04/07/2018). 
[23] Moon, M. (2015) 'Oculus program makes it possible for novices to animate VR

characters', Engadget [en línia]. Disponible a: https://www.engadget.com/2015/05/08/oculus-razer-hydra-geppetto-vr-animation/ (Consultat el 04/07/2018).

[24] (2015) 'SteamVR'[en línia]. Disponible a: https://store.steampowered.com/steamvr (Consultat el 04/07/2018).

[25] (2015) 'Tech Times' [en línia]. Disponible a: https://www.techtimes.com/ (Consultat el 04/07/2018).

[26] Jones, N. (2015) 'Top 10 Mobile Technologies and Capabilities for 2015 and 2016', Gartner [en línia]. Disponible a: https://www.gartner.com/doc/2665315/top--mobile-technologies-capabilities (Consultat el 05/07/2018).

[27] Asendador, S. H. (2016) 'Seis tecnologías que cambiarán el mundo según Google', Expansión. Economía Digital [en línia]. Disponible a: http://www.expansion.com/economia-digital/innovacion/2016/06/11/575c094b22601d01628b 4611.html (Consultat el 25/04/2018).

[28] Alkhatib, H. et al. (2014) 'IEEE CS 2022 Report', IEEE [en línia]. Disponible a: http://lifesciences.ieee.org/article-archive/ieee-cs-2022-report/. (Consultat el 22/05/2018).

[29] Anderson, J. (2014) 'Digital Life in 2025', Pew Research Center [en línia]. Disponible a: http://www.pewinternet.org/2014/03/11/digital-life-in-2025/ (Consultat el 16/05/2018).

[30] Hopkins, B. (2016) 'Forrester's top emerging technologies to watch in 2017-2021', Forrester [en línia]. Disponible a: https://go.forrester.com/blogs/16-09-14-forresters_top_emerging_technologies_to_watch_20 17 2021/ (Consultat el 22/05/2018).

[31] Steele, B. (2016) 'WSJ: Google is working on a standalone VR headset, too', Engadget [en línia]. Disponible a: https://www.engadget.com/2016/02/11/google-standalone-vr-headset/ (Consultat el 17/07/2018).

[32] Robertson, A. (2016) 'Google adds better audio to Cardboard's virtual reality', The Verge [en línia]. Disponible a: http://www.theverge.com/2016/1/13/10755526/google-cardboard-vr-spatial-audio-update (Consultat el 17/07/2018).

[33] Vincent, J. (2016) 'Nintendo is exploring VR again, 20 years after the Virtual Boy flop', The Verge [en línia]. Disponible a: http://www.theverge.com/2016/2/2/10893266/nintendo-virtual-reality-vr-return (Consultat el 17/07/2018).

[34] Machkovech, S. (2016) 'HTC Vive Pre impressions: A great VR system has only gotten better', Ars Technica [en línia]. Disponible a: https://arstechnica.com/gaming/2016/01/htc-vive-pre-impressions-a-great-vr-system-has-onl y-gotten-better/ (Consultat el 17/07/2018). 
[35] (2016) 'Augmented Reality. Home' Meta Company [en línia]. Disponible a: https://www.metavision.com/ (Consultat el 17/07/2018).

[36] Ziegler, C. (2016) 'Touchless haptic feedback completely freaked me out at CES', The Verge [en línia]. Disponible a: http://www.theverge.com/2016/1/8/10738792/harman-touchless-ultrasonic-haptic-feedback-c es-2016 (Consultat el 17/07/2018).

[37] Fingas, J. (2016) 'Google's virtual reality field trips are available to everyone' Engadget [en línia]. Disponible a: https://www.engadget.com/2016/06/27/google-opens-vr-expeditions-to-everyone/ (Consultat el 17/07/2018).

[38] Tarantola, A. (2016) 'Twitter confirms it's launching an AR division with new hire', Engadget [en línia]. Disponible a: https://www.engadget.com/2016/06/28/twitter-confirms-it-s-launching-an-ar-division-with-new -hire/ (Consultat el 17/07/2018).

[39] Amadeo, R. (2016) 'Google and Qualcomm team up to make Project Tango easy on your CPU', Ars Technica [en línia]. Disponible a: https://arstechnica.com/gadgets/2016/06/google-and-qualcomm-team-up-to-make-project-ta ngo-easy-on-your-cpul (Consultat el 17/07/2018).

[40] Miller, P. (2016) 'Nvidia's foveated rendering tricks for VR could improve graphics and immersion', The Verge [en línia]. Disponible a: http://www.theverge.com/2016/7/22/12260430/nvidia-foveated-rendering-vr-graphics-smi-ey e-tracking-siggraph (Consultat el 17/07/2018).

[41] Hruska, J. (2016) 'Apple's Tim Cook declares augmented reality a 'core technology' for the company', ExtremeTech [en línia]. Disponible a: http://www.extremetech.com/computing/233735-apples-tim-cook-declares-augmented-reality -a-core-technology-for-the-company (Consultat el 17/07/2018).

[42] Kastrenakes, J. (2016) 'Apple continues hiring augmented and virtual reality experts', The Verge [en línia]. Disponible a: http://www.theverge.com/2016/9/17/12952802/apple-augmented-virtual-reality-hires

(Consultat el 17/07/2018).

[43] Conditt, J. (2016) 'Use your fingers to play in Vive's world with the Manus VR glove', Engadget [en línia]. Disponible a: https://www.engadget.com/2016/03/10/htc-vive-manus-vr-glove-dev-kit-pre-order/ (Consultat el 17/07/2018).

[44] Conditt, J. (2016) 'Hands-in with the gloves that virtual reality needs', Engadget [en línia]. Disponible a: https://www.engadget.com/2016/03/16/hands-in-with-the-gloves-that-virtual-reality-needs/ (Consultat el 17/07/2018).

[45] Ingraham, N. (2016) 'Sennheiser's 3D audio for VR feels totally natural,' Engadget [en línia].

Disponible

a: 
https://www.engadget.com/2016/03/15/sennheiser-3d-audio-demo-ambeo/ (Consultat el 17/07/2018).

[45b] Williams, A. (2016) 'Nokia Ozo: A 360。 3D VR camera out to populate the barren content desert', Engadget [en línia]. Disponible a: https://www.engadget.com/2016/03/15/sennheiser-3d-audio-demo-ambeo/ (Consultat el 17/07/2018).

[46] Dent, S. (2016) 'Microsoft's hand-tracking magic may lead to gamepad-free VR', Engadget [en línia]. Disponible a: https://www.engadget.com/2016/06/28/microsoft-hand-tracking-virtual-reality/ (Consultat el 17/07/2018).

[47] Bohn, D. (2016) 'Tim Cook: Virtual reality is not 'a niche' and 'has some interesting applications", The Verge [en línia]. Disponible a: http://www.theverge.com/2016/1/26/10836066/tim-cook-virtual-reality-apple-not-niche-intere sting-applications (Consultat el 17/07/2018).

[48] Velazco, C. (2015) 'Doctors use Google Cardboard to explore a heart, save a life', Engadget [en línia]. Disponible a: https://www.engadget.com/2015/12/29/google-cardboard-teegan-lexcen/ (Consultat el 17/07/2018).

[49]Steele, B. (2016) 'Ai Weiwei's recent London art exhibit is available in VR', Engadget [en línia]. Disponible a: https://www.engadget.com/2016/01/21/ai-weiwei-art-exhibit-in-vr/ . (Consultat el 17/07/2018).

[50] Ravenscraft, E. (2016) 'Public Speaking for Cardboard Helps You Practice Speaking to an Audience', Life Hacker [en línia]. Disponible a: http://lifehacker.com/public-speaking-for-cardboard-helps-you-practice-speaki-1757280530 (Consultat el 17/07/2018).

[51] Watercutter, A. (2016), 'You Can Watch Sundance's VR Films Right on Your Smartphone', Wired [en línia]. Disponible a: https://www.wired.com/2016/01/sundance-vr-app/ (Consultat el 17/07/2018).

[52] Volpe, J. (2016) 'Fox pushes virtual reality to the limit with 30 minutes on Mars', Engadget [en línia]. Disponible a: https://www.engadget.com/2016/01/08/the-martian-vr-fox-interview/ $\quad$ (Consultat el 17/07/2018).

[53] Fisk, J. (2016) 'I Did Standup in Virtual Reality', Motherboard [en línia]. Disponible a: https://motherboard.vice.com/enlus/article/i-did-standup-in-virtual-reality (Consultat el 17/07/2018).

[54] Puerto, K. (2016) 'Ikea utiliza la realidad virtual de HTC Vive para enseñarte la cocina antes de comprarla', Xataka [en línia]. Disponible a: https://www.xataka.com/realidad-virtual-aumentada/ikea-utiliza-la-realidad-virtual-de-htc-vive -para-ensenarte-la-cocina-antes-de-comprarla (Consultat el 17/07/2018). 
[55] Nutt, C. (2016) ' Microsoft demos 'holoportation' 3D presence tech with HoloLens', Gamasutra [en línia]. Disponible a: http://www.gamasutra.com/view/news/268997/Microsoft $\{\backslash$ \}demos $\{\backslash\}$ holoportation $\{1\}$ \}D $\{\backslash\}$ presence $\{\backslash$ \}tech\{\\} with\{\\}HoloLens.php (Consultat el 17/07/2018).

[56] Gitlin, J. M. (2016) 'KTM brings the service manual into the 21st century with augmented reality', Ars Technica [en línia]. Disponible a: https://arstechnica.com/cars/2016/03/ktm-brings-the-service-manual-into-the-21st-century-wi th-augmented-reality/ (Consultat el 17/07/2018).

[57] Volpe, J. (2016) 'With HoloLens, you can now remodel your kitchen at Lowe's' Engadget [en línia]. Disponible a: https://www.engadget.com/2016/03/18/with-hololens-you-can-now-remodel-your-kitchen-at-l owes/ (Consultat el 17/07/2018).

[58] Alvarez, E. (2016) 'A VR experience transported me to a live soccer game', Engadget [en línia]. Disponible a: https://www.engadget.com/2016/03/17/virtually-live-virtual-reality-live-sports-gdc-2016/ (Consultat el 17/07/2018).

[59] (2016) 'El Barcelona vs. Real Madrid se emitirá en realidad virtual', La Vanguardia [en línia].

Disponible

a:

http://www.lavanguardia.com/television/20160401/40800983747/barsa-madrid-realidad-virtu al.html (Consultat el 17/07/2018).

[60] Steele, B. (2016) 'USA Today announces VR news show 'VRtually There', Engadget [en línia]. Disponible a: https://www.engadget.com/2016/03/10/usa-today-vrtually-there-vr-news-show/ (Consultat el 17/07/2018).

[61] Seppala,T. J. (2016) 'Watch astronauts make outer space Skype calls with HoloLens', Engadget [en línia]. Disponible a: https://www.engadget.com/2016/03/11/iss-skype-call-video/ (Consultat el 17/07/2018).

[62] Statt, N. (2016) 'Boeing is using Google Glass to build airplanes', The Verge [en línia]. Disponible

a:

http://www.theverge.com/2016/7/14/12189574/boeing-google-glass-ar-building-airplane-part $\underline{s}$ (Consultat el 17/07/2018).

[63] (2016) 'Beginning VR Development. Just A/VR Show', Channel 9 [en línia]. Disponible a: https://channel9.msdn.com/blogs/misslivirose/Beginning-VR-Development (Consultat el 17/07/2018).

[64] Lumb, D. (2016) 'Google opens Daydream VR platform to developers', Engadget [en línia].

Disponible

a:

https://www.engadget.com/2016/09/22/google-opens-daydream-vr-platform-to-developers/ (Consultat el 17/07/2018).

[65] Buckley, S. (2016) 'Razer's second OSVR devkit is now available for pre-order', Engadget len línia]. Disponible a: 
https://www.engadget.com/2016/07/20/razers-second-osvr-devkit-is-now-available-for-pre-or der/ (Consultat el 17/07/2018).

[66] Cardinal, D. (2016) '2016 is turning out to be an amazing year for augmented reality', ExtremeTech [en línia]. Disponible a: http://www.extremetech.com/extreme/224618-2016-is-turning-out-to-be-an-amazing-year-for -augmented-reality (Consultat el 17/07/2018).

[67] Cox, J. (2016) 'This year's Coachella attendees are being given custom Cardboard VR headsets', The Verge [en línia]. Disponible a: http://www.theverge.com/2016/3/9/11186360/coachella-google-cardboard-vr-headsets-welco me-box (Consultat el 17/07/2018).

[68] Bishop, B. (2016) 'The year that virtual reality took over Comic-Con', The Verge [en línia].

Disponible

a:

http://www.theverge.com/2016/7/25/12279274/virtual-reality-x-men-mr-robot-suicide-squad-c omic-con-2016 (Consultat el 17/07/2018).

[69] Davis, N. (2016) 'Long-term effects of virtual reality use need more research, say scientists', The Guardian [en línia]. Disponible a: https://www.theguardian.com/technology/2016/mar/19/long-term-effects-of-virtual-reality-use -need-more-research-say-scientists (Consultat el 17/07/2018).

[70] Lawler, R. (2016) 'Oculus Social launches on Gear VR, Facebook tie-in coming soon', Engadget [en línia]. Disponible a: https://www.engadget.com/2016/03/09/oculus-social-launches-on-gear-vr-facebook-tie-in-co ming-soon/ (Consultat el 17/07/2018).

[71] Santa Maria, C. (2016) 'The incredible future of VR isn't video games or movies, it's medicine', Splinter [en línia]. Disponible a: https://splinternews.com/the-incredible-future-of-vr-isnt-video-games-or-movies-1793855265 (Consultat el 17/07/2018).

[72] Orland, K. (2016) 'Epic looks outside of gaming for new uses of Unreal Engine', Ars Technica [en línia]. Disponible a: https://arstechnica.com/gaming/2016/03/epic-looks-outside-of-gaming-for-new-uses-of-unrea l-engine/ (Consultat el 17/07/2018).

[73] Mavim, (2017) Gartner Top 10 Technology Trends 2017 [video en línia]. Disponible a: https://www.youtube.com/watch?v=fTvFO-2LtNM (Consultat el 19/07/2018).

[74] Gatherer, A. (2017) 'Ten Communications Technology Trends for 2017', IEEE Communications Society len línia]. Disponible a: http://www.comsoc.org/ctn/ten-communications-technology-trends-2017 (Consultat el 19/07/2018).

[75] 'Realitat mixta' (2018) Wikipedia [en línia]. Disponible a: https://ca.wikipedia.org/wiki/Realitat mixta (Consultat el 19/07/2018).

[76] Clarisó R., et al. (2017) 'Informe de l'Observatori Tecnològic dels Estudis d'Informàtica, Multimèdia i Telecomunicació', Estudis d'Informàtica, Multimèdia i Telecomunicació, Universitat Oberta de Catalunya [en línia]. Disponible a: 
http://openaccess.uoc.edu/webapps/o2/bitstream/10609/80806/6/informeobservatori2017pu blic.pdf (Consultat el 19/07/2018).

[76b] Ibídem.

[77] Cannon, T. (2017) 'How Virtual Reality could transform Higher Education', Red Brick Research [en línia]. Disponible a: https://www.redbrickresearch.com/2017/08/30/how-virtual-reality-could-transform-higher-edu cation/ (Consultat el 26/07/2018).

[78] Durlach, N. I. et al. (1992) Virtual environment technology for training (VETT), The Virtual Environment and Teleoperator Research Consortium (VETREC). Bolt, Beranek, and Newman Report, 7661 [en línia]. Disponible a: https://pdfs.semanticscholar.org/0bc8/480093caac76dd238ece849292552475a7b8.pdf

(Consultat el 13/11/2018).

[79] Mann, E. (2016) 'Is virtual reality ready for school?', Brookings [en línia]. Disponible a: https://www.brookings.edu/blog/brown-center-chalkboard/2016/09/08/is-virtual-reality-ready-f or-school-2/ (Consultat el 26/07/2018).

[80] Major, E. (2017) '4 Ways to Use Augmented and Virtual Reality Apps in the Classroom', Common Sense [en línia]. Disponible a: https://www.commonsense.org/education/blog/4-ways-to-use-augmented-and-virtual-realityapps-in-the-classroom (Consultat el 26/07/2018).

[81] Metry, M. (2017) 'Best 15+ Free Virtual Reality Apps for Education', VU Dream [en línia]. Disponible a: http://www.vudream.com/best-15-free-virtual-reality-apps-for-education/ (Consultat el 26/07/2018).

[82] Goode, L. (2017) 'Hugo Barra left Xiaomi to run VR at Facebook', The Verge, 2017. [en línia].

Disponible

a:

http://www. theverge.com/2017/1/25/14395062/hugo-barra-left-xiaomi-to-run-vr-at-facebookoculus (Consultat el 26/07/2018).

[83] Savov, V. (2017) 'HTC's top Vive designer is leaving to work on Google Daydream', The Verge [en línia]. Disponible a: http://www.theverge.com/2017/1/26/14395134/google-daydream-claude-zellweger-htc-vive-d esigner (Consultat el 26/07/2018).

[84] Robertson, A. (2017) 'Google isn't building the ultimate VR headset. It's setting the foundation for one', The Verge [en línia]. Disponible a: https://www.theverge.com/2017/5/18/15657138/google-io-2017-standalone-vr-headset-daydr eam-update (Consultat el 26/07/2018).

[85] (2017) 'Samsung triplica los píxeles en su nueva pantalla para gafas VR', Omicrono. [en línia]. Disponible a: http://omicrono.elespanol.com/2017/06/pantalla-vr-samsung-alta-resolucion/ (Consultat el 26/07/2018).

[86] Orland, K. (2017) 'Valve's Gabe Newell: VR could 'turn out to be a complete failure', Ars Technica [en línia].

Disponible a: 
https://arstechnica.com/gaming/2017/02/valves-gabe-newell-vr-could-turn-out-to-be-a-compl ete-failure/ (Consultat el 26/07/2018).

[87] Archanco, E. (2017) 'Apple le tira el guante a Palmer Luckey a la cara: los nuevos iMac se meriendan la realidad virtual', Apple Sfera [en línia]. Disponible a: https://m.applesfera.com/sobremesa/apple-le-tira-el-guante-a-palmer-luckey-a-la-cara-los-nu evos-imac-se-meriendan-la-realidad-virtual (Consultat el 26/07/2018).

[88] Muela, C. (2017) 'Google Glass Enterprise 2017, características, precio y aplicaciones que usar', Xataka [en línia]. Disponible a: https://m.xataka.com/realidad-virtual-aumentada/no-estaban-muertas-google-glass-enterpris e-salen-a-la-venta-y-para-esto-sirven-en-2017 (Consultat el 26/07/2018).

[89] Martí, A. (2017) 'Sentir frío, calor y dolor al jugar con realidad virtual: así es como ThermoReal pretende mejorar la experiencia', Xataka [en línia]. Disponible a: https://m.xataka.com/realidad-virtual-aumentada/sentir-frio-calor-y-dolor-al-jugar-con-realida d-virtual-asi-es-como-thermoreal-pretende-mejorar-la-experiencia. (Consultat el 26/07/2018). [90] Barnes, B. (2017) 'Virtual Reality's Potential for Magic Gets Real', The New York Times [en línia]. Disponible a: https://www.nytimes.com/2017/02/19/business/media/void-start-up-virtual-reality.html? $r=1$. (Consultat el 26/07/2018).

[91] McAloon, A. (2017) 'Analyst: 1M VR headsets shipped last', Gamasutra [en línia]. Disponible

a: https://www.gamasutra.com/view/news/310564/Analyst_1M_VR_headsets_shipped_las\qu arter_an_industry_first.php (Consultat el 26/07/2018).

[92] (2017) 'Pilot both holographic and real drones with these glasses', Futurism [en línia]. Disponible a: https://twitter.com/futurism/status/932413205317435395 (Consultat el 26/07/2018).

[93] (2017) 'Una aerolínea usa gafas de realidad virtual para sacar más información de los pasajeros', 20 minutos [en línia]. Disponible a: http://www.20minutos.es/noticia/3048624/0/aerolinea-gafas-realidad-virtual-informacion-pas ajeros/ (Consultat el 26/07/2018).

[94] (2017) 'Virtway, líder en usuarios simultáneos con su mundo virtual 3D para móviles', Parcela Digital' [en línia]. Disponible a: http://parceladigital.com/2017/02/14/virtway-lider-en-usuarios-simultaneos-con-su-mundo-virt ual-3d-para-moviles-the-education-district/ (Consultat el 26/07/2018).

[95] (2017) 'Crearon una app de realidad virtual para recorrer el interior de un perro', El Día [en línia]. Disponible a: http://www.eldia.com/nota/2017-6-26-19-43-32-crearon-una-app-de-realidad-virtual-para-rec orrer-el-interior-de-un-perro-informacion-general (Consultat el 26/07/2018).

[96] Álvarez, R. (2017) 'De forma sorpresiva Nintendo anuncia su primer juego de realidad virtual para HTC Vive: "Mario Kart Arcade GP VR"', Xataka [en línia]. Disponible a: 
https://m.xataka.com/videojuegos/de-forma-sorpresiva-nintendo-anuncia-su-primer-juego-de -realidad-virtual-para-htc-vive-mario-kart-arcade-gp-vr (Consultat el 26/07/2018).

[97] Kwatra, V., Frueh C. (2017) 'Research Blog: Headset 'Removal' for Virtual and Mixed Reality', Google Al Blog [en línia]. Disponible a: https://research.googleblog.com/2017/02/headset-removal-for-virtual-and-mixed.html?m=1 . (Consultat el 26/07/2018).

[97b] Orland, K. (2017) 'Valve's Gabe Newell: VR could 'turn out to be a complete failure", Ars Technica len línia]. Disponible a: https://arstechnica.com/gaming/2017/02/valves-gabe-newell-vr-could-turn-out-to-be-a-compl ete-failure/ (Consultat el 07/11/2018).

[98] Sabán, A. (2017) 'Google lanza Blocks para potenciar la creación de contenido en realidad virtual', Hipertextual [en línia]. Disponible a: https://hipertextual.com/2017/07/blocks-google-realidad-virtual (Consultat el 26/07/2018).

[99] Gisbert Ponsola, J. (2017) 'Facebook ahora permitirá transmitir en vivo desde su aplicación de realidad virtual', Andro 4 all [en línia]. Disponible a: https://andro4all.com/2017/07/retransmitir-directo-facebook-vr?utm source=feed-Andro4all\& utm medium=rss (Consultat el 26/07/2018).

[100] (2018) 'Oculus Research to Present Focal Surface Display Discovery at SIGGRAPH',

Oculus [en línia]. Disponible a:
https://www.oculus.com/blog/oculus-research-to-present-focal-surface-display-discovery-at-s iggraph/ . (Consultat el 26/07/2018).

[102] (2017) 'Holograms: The future of near-eye display?', Microsoft Research [en línia]. Disponible

a:

https://www.microsoft.com/en-us/research/blog/holograms-future-near-eye-display/

(Consultat el 26/07/2018).

[103] Robertson, A. (2017) 'Nvidia just unveiled the millionth VR "Holodeck" and it's for looking at cars', The Verge [en línia]. Disponible a: https://www.theverge.com/2017/5/10/15613018/nvidia-vr-project-holodeck-koenigsegg-car-d emo (Consultat el 26/07/2018).

[104] Robertson, A. (2017) 'The HTC Vive will track eye movement with a \$220 upgrade kit', The Verge [en línia]. Disponible a: https://www.theverge.com/2017/5/1/15503932/htc-vive-x-7invensun-aglass-eye-tracking-upg rade (Consultat el 26/07/2018).

[105] Fingas, J. (2017) 'Munich ends its long-running love affair with Linux', Engadget [en línia].

Disponible

a: https://www.engadget.com/2017/11/26/linux-pioneer-munich-switches-to-windows/

(Consultat el 26/07/2018).

[106] Ong, T. (2017) 'Nokia is killing off its Ozo virtual reality camera and cutting 310 jobs', The Verge [en línia]. Disponible a: 
https://www.theverge.com/2017/10/10/16452114/nokia-killing-off-ozo-virtual-reality-camera-3 10-jobs-lost (Consultat el 26/07/2018).

[107] Warren, T. (2017) 'I tried Microsoft's first Mixed Reality headset and it was just like VR', The Verge [en línia]. Disponible a: https://www.theverge.com/2017/5/11/15627374/microsoft-windows-mixed-reality-hands-on (Consultat el 26/07/2018).

[108] Machkovech, S. (2017) 'Microsoft's new VR controllers will be great until SteamVR "Knuckles" arrive', Ars Technica [en línia]. Disponible a: https://arstechnica.com/gaming/2017/05/microsofts-new-vr-controllers-will-be-great-until-stea mvr-knuckles-arrive/ (Consultat el 26/07/2018).

[109] Hardawar, D. (2017) 'I finally believe in Microsoft's mixed reality vision', Engadget [en línia]. Disponible a: https://www.engadget.com/2017/05/12/microsoft-hololens-mixed-reality/ (Consultat el 26/07/2018).

[110] Hills-Duty, R. (2017) 'Chinese School Opens Full-Function VR Classrooms', VRFocus [en línia].

Disponible

a:

https://www.vrfocus.com/2017/10/chinese-school-opens-full-function-vr-classrooms/ (Consultat el 26/07/2018).

[111] Warren, T. (2017) 'Watch Microsoft demo and explain Windows Mixed Reality', The Verge [en línia]. Disponible a: https://www.theverge.com/2017/10/4/16417652/microsoft-windows-mixed-reality-demo-keyn ote (Consultat el 26/07/2018).

[112] (2017) 'Microsoft y Samsung colaboran en el desarrollo de un casco de realidad mixta', Europa Press [en línia]. Disponible a: http://www.europapress.es/portaltic/gadgets/noticia-microsoft-samsung-colaboran-desarrollo -casco-realidad-mixta-20170927125506.html (Consultat el 26/07/2018).

[113] Robertson, A. (2017) 'Intel is cutting plans for its Project Alloy "merged reality" headset', The Verge len línia]. Disponible a: https://www.theverge.com/2017/9/22/16351900/intel-project-alloy-vr-merged-reality-headsetreference-design-discontinued (Consultat el 26/07/2018).

[114] 'Idealisme subjectiu' (2018) Viquipèdia [en línia]. Disponible a: https://ca.wikipedia.org/wiki/ldealisme_subjectiu (Consultat el 04/09/2018).

[115] (2018) 'Inventor in the field of Virtual Reality. Telesphere Mask', Morton Heilig [en línia]. Disponible a: http://www.mortonheilig.com/InventorVR.html (Consultat el 04/09/2018).

[116] Ibídem. (Patent número 2,955,156 del 4 d'octubre de 1940. Document disponible a http://www.mortonheilig.com/TelesphereMask.pdf).

[117] 'History of Virtual Reality', Virtual Reality Society, 2017. [en línia]. Disponible a: https://www.vrs.org.uk/virtual-reality/history.html. (Consultat el 04/09/2018).

[118] Medienkunstnetz.de. Myron Krueger. Videoplace [en línia]. Disponible a: http://www.medienkunstnetz.de/works/videoplace/images/1/ (Consultat el 04/09/2018). 
[119] Jaronlanier.com. VPL Software Lives! [en línia]. Disponible a: http://www.jaronlanier.com/vpl.html (Consultat el 04/09/2018).

[120] Evl.uic.edu. Sayre Glove (first wired glove) [en línia]. Disponible a: https://www.evl.uic.edu/entry.php?id=2162 (Consultat el 05/09/2018).

[121] Sorene, P., (2014) 'Jaron Lanier's EyePhone: Head And Glove Virtual Reality In The 1980s', Flashbak [en línia]. Disponible a: https://flashbak.com/jaron-laniers-eyephone-head-and-glove-virtual-reality-in-the-1980s-261 80/ (Consultat el 05/09/2018).

[122] (2017) 'VPL Research Jaron Lanier', Virtual Reality Society [en línia]. Disponible a: https://www.vrs.org.uk/virtual-reality-profiles/vpl-research.html (Consultat el 05/09/2018).

[123] Lamble, R. (2013) 'The 1983 videogame crash: what went wrong, and could it happen again?', Den of Geek [en línia]. Disponible a: http://www.denofgeek.com/games/24531/the-1983-videogame-crash-what-went-wrong-andcould-it-happen-again (Consultat el 05/09/2018).

[124] Segaretro.org. Sega VR [en línia]. Disponible a: https://segaretro.org/Sega_VR (Consultat el 05/09/2018).

[125] Fowle, K. (2015) 'A look back at the doomed virtual reality boom of the 90s', Kill Screen [en línia]. Disponible a: https://killscreen.com/articles/failure-launch/ (Consultat el 05/09/2018).

[126] Silicon Classics, (2012) Forte VFX1 Virtual Reality Headgear Review (1995) [Vídeo en línia]. Disponible a https://www.youtube.com/watch?v=J0n5B3fl-bU (Consultat el 05/09/2018).

[127] Veure nota 117.

[128] Wikia. Second Life. The Rig [en línia]. Disponible a: http://secondlife.wikia.com/wiki/The Rig (Consultat el 07/09/2018).

[129] Robertson, A., Zelenko, M. (comp.) (2018) 'Voices form a virtual past. An oral history of a technology whose time has come again', The Verge [en línia]. Disponible a: https://www.theverge.com/a/virtual-reality/oral history (Consultat el 07/09/2018).

[130] Gonzales, D., Criswell, D., Heer, E. (1991) 'Automation and Robotics for the Space Exploration Initiative: Results from Project Outreach', Rand [en línia]. Disponible a https://www.rand.org/content/dam/rand/pubs/notes/2009/N3284.pdf. Citat a 'Virtual Reality' (2018) Wikipedia [en línia]. Disponible a: https://en.wikipedia.org/wiki/Virtual_reality\#cite_note-23 (Consultat el 06/09/2018).

[131] Veure referència $\underline{129}$.

[132] lbídem.

[133] Etc.cmu.edu. Entertainment Technology Center [en línia]. Disponible a: https://www.etc.cmu.edu/learn/about-the-etc/ (Consultat el 14/09/2018).

[134] Rodríguez García, E. (2017) 'Las HoloLens ya están disponibles en España', Omicrono [en línia]. Disponible a: 
https://omicrono.elespanol.com/2017/12/microsoft-hololens-ya-en-espana/ (Consultat el 06/11/2018).

[135] Wahab, A. (2017) 'Augmented Reality. A New Dawn of Possibilities in Online Training/eLearning', Swift elearning [en línia]. Disponible a: http://www.swiftelearningservices.com/augmented-reality-a-new-dawn-of-possibilities-in-onli ne-training-elearning/ (Consultat el 06/11/2018).

[136] Donachie, P. (2017) 'Teacher ed programs turn to virtual reality', Education Dive [en línia].

https://www.educationdive.com/news/teacher-ed-programs-turn-to-virtual-reality/511608/ (Consultat el 06/11/2018).

[137] Howell, E. (2016) 'Aliens Attack! Space Station Astronauts Fight VR Invasion with HoloLens', Space.com, [en línia]. Disponible a: https://www.space.com/32802-space-invaders-on-station-virtual-reality.html (Consultat el 07/11/2018). 